\title{
Combination of Local, Global and k-mean using Wavelet Transform for Content Base Image Retrieval
}

\author{
Ekta Gupta and Rajendra Singh Kushwah \\ Department of Computer Science \& Engineering \\ Institute of Technology \& Management \\ Gwalior-India \\ ektagupta308@gmail.com,rajendrasingh.ind@rediffmail.com
}

\begin{abstract}
In today's time, the requirement of content based image retrieval technique is more and more because of diverse areas such as Data Mining, Remote Sensing and Management of Earth Resources, , Crime Prevention, Weather forecasting, E-commerce, Medical Imaging. The proposed paper presents the content based image retrieval, using features like color and texture, called WBCHIR (Wavelet Based Color Histogram Image Retrieval).The shape and shade features are extracted in the course of wavelet transformation and color histogram and the arrangement of these features is vigorous to scaling and conversion of objects in an image. It is the first time to present segmentation and grid, feature extraction, K-means module and k-nearest neighbor clustering algorithms and bring in the neighborhood module to build the CBIR system. It is the hybrid method of global and local features with k-means clustering algorithm.
\end{abstract}

\section{Keywords: CBIR, K-means, DWT, Global Feature}

\section{Introduction}

Due to rapid progress in the Internet, and the obtain ability of image taking devices such as image scanners, digital cameras, the size of digital image collection is increasing rapidly. CBIR is a technique which uses chromatic features of an image such as color, texture, shape to search images from an enormous database similar to user's query. The CBIR system have been used in variety applications such as Fashion, Engineering design and Interior design, Journalism and Web Advertising, Medical diagnosis, Architectural, and Crime prevention etc. In the past decade, many image retrieval systems have been effectively developed, such as the IBM QBIC [1] System, developed at the IBM Alma den Research Center, the VIRAGO System [2], developed by the Vi rage Incorporation, the Photo book System [3], developed by the MIT Media Lab, the Visual Seek System [4], developed at Columbia University, the WBIIS System [5] advanced at Stanford University, and the Blob-world System [6], advanced at U.C. Berkeley and Simplicity System [7]. There are three fundamental bases for Content Based Image Retrieval which are multidimensional indexing, retrieval system design and visual feature extraction. The color feature can be achieved by the techniques like averaging and histograms. The texture feature can be grasped by using transforms or vector quantization. The shape feature can be reached by using gradient operators or morphological operators. The color histogram for color feature and wavelet demonstration for texture and location info of an image. This decreases the handling time for retrieval of an image with more talented legislatures. The abstraction of color features from digital images depends on an understanding of the theory of color and the representation of color in digital images. Color spaces are a main essential for relating color to its representation in digital form. This work texture and color features are discovered as a means of image content description. Image classification is performed in according to global features for 
describing the texture and color content for the whole image. It could be also possible to extract the same features for previously segmented objects. Feature extraction is the first step of content-based image retrieval. There are a number of different ways to extract. Feature currently in use in terms of technology. Most major feature extraction methods fall into color, texture, shape or spatial relations. Color feature is a global feature which cannot be expressed as rich regional object details. Texture feature is susceptible to illumination changes and affine. When the object rotates or scales or transforms, shape feature and spatial relations feature will not capture the accurate feature. In this paper we suggest a hybrid method for CBIR that takes into account global, local and k-mean features an image. Towards this, Stationary Wavelet Transform (SWT) is applied on input image to extract horizontal, vertical and diagonal detail matrices. SWT is used because of its translational invariant stuff. After this global textural features are mined using Gray level Co-occurrence Matrix for each of these sub-matrices. To help the retrieval process, a local descriptor is also computed by splitting the image into sub-regions. Finally Euclidean distance is used to retrieve the relevant results given sample of unknown class into one of $\mathrm{n}$ classes. To design an real algorithm for texture organization, it is essential to find a set of texture features with good discriminating power. The wavelet methods offer computational benefits over other methods for texture classification.

A mutual method in texture analysis involves the calculation of GLCM as a secondorder texture amount [21]. GLCM defines the frequency of one gray tone seeming in a specified spatial linear association with another gray tone, within the area under study. Several statistical constraints can be extracted from the GLCM. Some of these constraints are related to specific first-order statistical concepts, such as variance and contrast, and have a clear textural meaning. Other parameters contain textural information but are connected with additional than one specific textural meaning.

Six textural constraints are reflected: energy, inverse difference moment, variance, correlation, entropy and contrast. The aim of this study was to appraise functional differences among these six constraints and to subordinate a textural meaning to each constraint, in order to perform an image-independent feature selection among them. Wavelet transforms can reduce the dimensions of images and allow good resolution in time and frequency, and multi-resolution techniques can be used in wavelet transforms, so the method that color feature is extracted from low frequency band of wavelet transform and texture feature from high frequency bands is not only simple and quick to implement but also can lead to good retrieval results. Color feature is one of the most important lowlevel visual features, so color index is one of the basic methods of image retrieval. Texture feature responds to the detail information of local image and measure the relation of pixels in local regions, so texture feature is also very important. Only one of the two features is used in most former retrieval systems, those of which only using color feature ignore the local details, and those only using texture feature ignore the general color information, so these systems cannot give satisfactory results. To solve this kind of question, a method using both color and texture feature in our retrieval system is proposed in this paper. In this system, the weights of color similarity and texture similarity are determined self-adaptively by the classification of texture image and non-texture image, and the weights are adjusted by relevance feedback so that the retrieval results accord with the user's retrieval goal gradually.

\section{Literature Review}

Literature survey is important for sympathetic and gaining more knowledge about a precise area of the subject. Towards this a brief overview of the different existing techniques for CBIR is presented. Before CBIR, the traditional image retrieval is usually based on text. Text based image retrieval has been discussed over those years. The textbased retrieval is easy to find out some disadvantages such as: 
1. Manually gloss is always involved by human's feeling, state, etc. which directly results in what is in the images and what is it about.

2. Gloss is never complete.

3. Linguistic and philosophy difference always cause problems the same image is usually text out by many diverse ways.

4. Errors such as spelling error or spell difference leads to totally different effects.

In order to overcome these drawbacks, content based images retrieval (CBIR) was first presented by Kato in 1992. Lin et al. Proposed a color-texture and color-histogram based image retrieval system (CTCHIR). They proposed three image features, based on color, texture and shape, as color co-occurrence matrix (CCM), difference between pixels of scan pattern (DBPSP) and color histogram for K-mean (CHKM) respectively and a technique for image retrieval by mixing CCM, DBPSP and CHKM to enhance image detection rate and simplify deduction of image retrieval. From the experimental results they found that, their proposed method outstrips the Jhanwar et al. [9] and Hung and Dai [10] methods. Raghupathi et al. have made a comparative study on image retrieval techniques, using different feature extraction methods like color histogram, Gabor Transform, color histogram+ gab-our transform, Contour-let Transform and color histogram + contour-let transform. Hire-math and Pujari proposed CBIR system based on the color, texture and shape features by separating the image into tiles. In order to overcome these drawbacks, content based images retrieval (CBIR) was first introduced by Kato in 1992. Lin et al. [8] proposed a color-texture and color-histogram based image retrieval system (CTCHIR). They proposed three image features, based on color, texture and color, as color co-occurrence matrix (CCM), difference between pixels of scan pattern (DBPSP) and color histogram for K-mean (CHKM) respectively and a method for image retrieval by integrating CCM, DBPSP and CHKM to improve image discovery rate and simplify calculation of image retrieval. From the experimental results they found that, their proposed method outperforms the Jhanwar et al. [9] and Hung and Dai [10] methods. Raghupathi et al. have made a comparative study on image retrieval techniques, using different feature extraction methods like color histogram, Gabor Transform, color histogram+ gab-our transform, Contour-let Transform and color histogram + contour-let transform. Hire-math and Pujari [11] proposed CBIR system based on the color, texture and shape features by partitioning the image into tiles. The features computed on tiles serve as local descriptors of color and texture features. The color and texture analysis are analyzed by using two level grid frameworks and the shape feature is used by using Gradient Vector Flow. The comparison of experimental result of proposed method with other system [12-13] found that, their proposed retrieval system gives better performance than the others. Rao et al. [14] proposed CTDCIRS (color-texture and dominant color based image retrieval system), they combined three features like Motif co-occurrence matrix (MCM) and difference between pixels of scan pattern (DBPSP) which describes the texture features and dynamic dominant color (DDC) to extract color feature. They compared their results with the work of Jhanwar et al. [9] and Hung and Dai [10] and found that their method gives better retrieval results than others.

\section{Methodology}

Feature extraction is an important step in any CBIR system. The retrieval accuracy highly depends upon the extent the given feature vector represents the image under test. In our technique we have considered both local as well as global features. 


\section{A. Computation of Global Features}

This method deals with image worldwide and tries to differentiate it by using visual/statistical features calculated from the entire image. Visual features are divided into three features: primitive features such as shade or figure rational skin texture such as identity of objects shown and abstract features such as significance of scenes depicted.

\section{1) Color:}

In domain of photograph retrieval, color has been the most efficient feature and almost all systems employ colors. There are three colors in image are red, green, blue (RGB) color space. Color histograms are used to match up to images in many applications. The main advantages are effectiveness, and insensitivity to small changes in camera viewpoint. However, color histograms need spatial information.

\section{2) Texture:}

Some of the most shared measures for taking the texture of images are wavelets and Gabor filters. These texture events try to imprisonment the characteristics of the image or image parts with respect to changes in certain directions and the scale of the changes. This is most valuable for regions or images with similar texture. Again, invariances with admiration to rotations of the image, shifts or scale changes can be included into the feature space. Other widespread texture descriptors contain features derived from cooccurrence matrices, features based on the factors of the Fourier transform and the socalled World features [15].

\section{3) Shape features:}

There are many shape representation and description techniques in the literature. Marr and Nishihara [16] and Braddy [17] have thoroughly discussed representation and sets of criteria for the evaluation of shape. Shape description or representation is an important issue both in object recognition and classification. It has been used in CBIR in conjunction with color and other features for indexing and retrieval. Many methods described chain code, polygonal approximations, curvature, Fourier descriptors and moment descriptors have been proposed and used in various applications [18]. The query images are represented by Fourier descriptors which serve powerful boundary-shape representation tools because of invariance property in affine transformation. Among the well-known shape descriptors, the Zernike moments have been successfully used in many shape contests [19].

\section{I) Stationary Wavelet Transformation}

Discrete wavelet transformation (DWT) is used to transform an image from spatial domain into frequency domain [23-25]. The wavelet transform signifies a function as a superposition of a family of basic functions called wavelets. Wavelet transforms extract information from signal at different scales by passing the signal through low pass and high pass filters. Wavelets provide multi resolution capability and good energy compaction. Wavelets are robust with respect to color intensity shifts and can capture both texture and shape information efficiently. The wavelet transforms can be computed linearly with time and thus allowing for very fast algorithms. DWT decomposes [26] a signal into a set of Basis Functions and Wavelet Functions. The wavelet transform calculation of a two-dimensional image is also a multi-resolution approach, which applies recursive filtering and sub-sampling. At each level (scale), the image is decomposed into four frequency sub-bands, LL, LH, HL, and HH where L denotes low frequency and $\mathrm{H}$ denotes high frequency as shown in Figure1. 


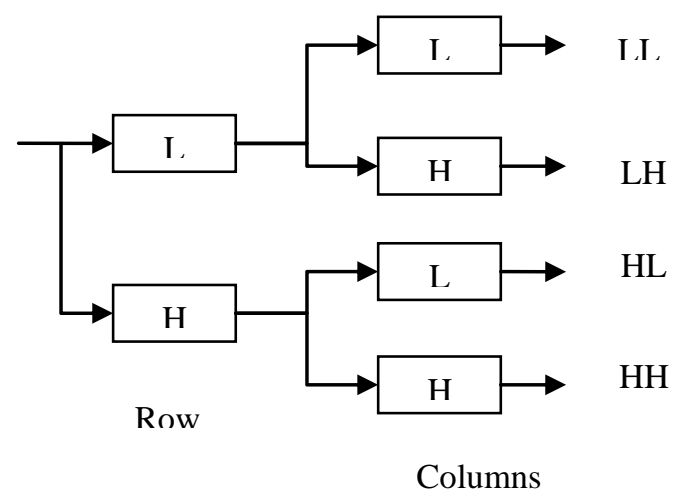

Figure 1. Stationary Wavelet Decomposition of a Two-dimensional Image

$$
\begin{aligned}
& L L_{j+1}(x, y)=\sum_{m, n} L[n] L[m] L L_{j\left(2^{j+1} m-x, 2^{j+1} n-y\right)} \\
& L H_{j+1}(x, y)=\sum_{m, n} L[n] H[m] L L_{j\left(2^{j+1} m-x, 2^{j+1} n-y\right)} \\
& H L_{j+1}(x, y)=\sum_{m, n}^{m, n} H[n] L[m] L L_{j\left(2^{j+1} m-x, 2^{j+1} n-y\right)} \\
& H H_{j+1}(x, y)=\sum_{m, n}^{m, n} H[n] H[m] L L_{j\left(2^{j+1} m-x, 2^{j+1} n-y\right)}
\end{aligned}
$$

These sub-band images would have the same size as that of original image because no down sampling is performed during the wavelet transformation. In our approach we have used "Haar" wavelet to perform multi-layer stationary wavelet decomposition on the input 2D image. Mathematically, the wavelet decomposition can be described.

\section{II) Gray Level Co-occurrence Matrix}

To reach this impartial, the GLCM constraints assessment is first carried out on a theoretic basis [22]. The reason for this choice is to overwhelmed a mutual limit which affects several articles, looked in the literature, dealing with the problem of the GLCM statistical constraints variety, where experimental results lack in general validity. Numerical examples should be provided, whenever essential, to support the theoretical conclusions. In this paper, no plots in 2-dimensional space are used as a assessable description of different GLCM parameters compassion for different types of texture [23]. This kind of analysis would have required the supervised selection of a combination of several texture types in order to stress some generic properties of the different texture parameters. This supervised process would have been very complex to implement. For this reason, an alternative strategy was developed to verify the theoretical deliberations. First, a test image presenting a wide variety of texture types was selected. Second, cluster analysis, adopted as an unsupervised investigation tool, was applied to the test image for each texture parameter in order to reveal its numerical behavior. Third, a qualitative (visual) comparison between the texture types in the original image and the cluster output image should confirm the validity of the general properties provided by the theoretical attentions. The computation of GLCM's requires an image partition stage. In order to reduce computation time, GLCM is assigned to the entire pixel block belonging to the GLCM computation segment and not to the individual pixel at the cell center. An initial image partition is made from $8 \times 8$ pixel segments. It must be stressed that GLCM computation must be performed within a realistic classification method. For instance, 
texture analysis should not be applied to those parts of the image that could be classified by a reliable non textural (e.g., spectral) investigation.

$$
\begin{gathered}
\text { ene }=\sum_{i=0}^{N_{g-1}} \sum_{j=0}^{N_{g-1}} g^{2}(i, j) \\
\text { con }=\sum_{i=0}^{N_{g-1}} \sum_{j=0}^{N_{g-1}}(i-j)^{2} g(i, j) \\
\text { cor }=\sum_{i=0}^{N_{g-1}} \sum_{j=0}^{N_{g-1}} \frac{(i-\mu)(j-\mu) g(i . j)}{\sigma^{2}} \\
i d m=\sum_{i=0}^{N_{g-1}} \sum_{j=0}^{N_{g-1}}\left[\frac{1}{\left(1+(i-j)^{2}\right)}\right] g(i, j)
\end{gathered}
$$

B. Computation of Local Features

A global descriptor uses the visual features of the whole image, while a local descriptor takes into account the regions or objects to describe the image. To compute local features we first divide the processed image into blocks and obtain a descriptor for each block. Figure 2 shows the method of splitting the image into three different subregions regions.

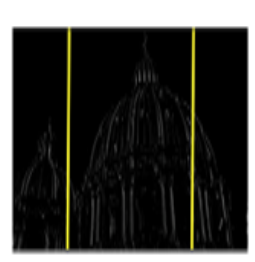

(a)

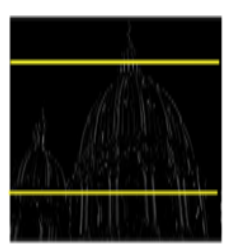

(b)

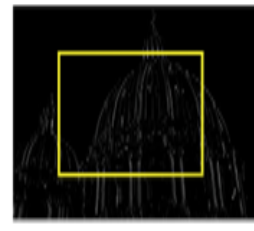

(c)

\section{Figure 2. Differennt Templates for Spliting Images into (a) Vertical Crop;(b)} Horizontal Crop;(c) Cenntral Crop

After splitting the image into sub-regions, two statistical measures are computed for each region. These measures are mean $(\mu)$ and standard deviation $(\sigma)$ [27].

$$
\begin{gathered}
\mu=\frac{\sum_{i=1}^{M} \sum_{j=1}^{M} f(i, j)}{M \times N} \\
\sigma=\sqrt{\frac{\sum_{i=1}^{M}(|f(i, j)|-\mu)^{2}}{M \times N}}
\end{gathered}
$$


Here $M, N$ represent the dimensions of the image and $f(i, j)$ represents the intensity value at an index $(i, j)$. Mean describes the average intensity over the image, while standard deviation shows amount of variation or dispersion from the mean value.

\section{Feature Vector Generation}

Having computed the local as well as global features [28] for the query image the next step is to combine all these features in a single feature vector which will be used for comparison during similarity matching. We obtain a total of 12 global features, 4 from each GLCM computed over horizontal, vertical and diagonal sub-matrices. Thus the dimension of global feature vector fG is $1 \times 12$ and is represented as in. In addition we consider 2 local measures for each region of image (Figure 2) yielding in total a 6 element local feature vector for each of horizontal, vertical and diagonal sub-matrices. The dimension of feature vector for each sub-matrix is $1 \times 6$. Hence the final dimensions of local feature vector fL will be $1 \times 18$ and is represented as in. The next step is to concatenate both the feature vectors to generate a single feature vector f Querry which will represent the query image. The dimensions of this main feature vector will be $1 \times 30$ and is represented as in. A similar feature vector fDataBase having identical dimensions to query image's feature vector is computed for every database image and is used during similarity matching process.

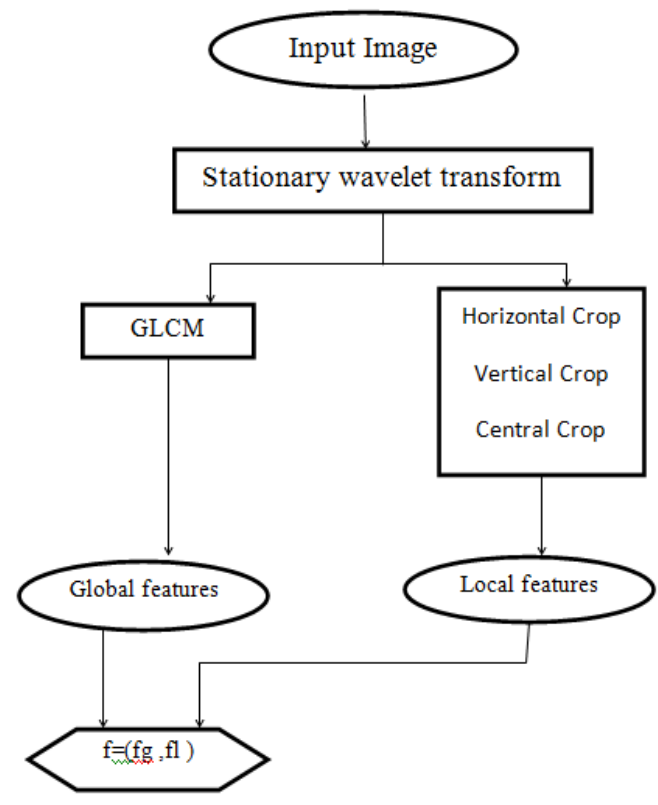

Figure 3. Schematic for Feature Vector Generation

$$
\begin{aligned}
& \mathrm{fG}=\left(\mathrm{f}_{\mathrm{GH}}, \mathrm{f}_{\mathrm{GV}}, \mathrm{f}_{\mathrm{GD}}\right) \\
& \mathrm{f}_{\mathrm{GH}}=\left(\mathrm{f}_{\mathrm{GHene}}, \mathrm{f}_{\mathrm{GHcor}}, \mathrm{f}_{\mathrm{GHcon}}, \mathrm{f}_{\mathrm{GHidm}}\right) \\
& \mathrm{f}_{\mathrm{GV}}=\left(\mathrm{f}_{\mathrm{GVene}}, \mathrm{f}_{\mathrm{GV} \text { cor }}, \mathrm{f}_{\mathrm{GV} \text { con }}, \mathrm{f}_{\mathrm{GVidm}}\right) \\
& \mathrm{f}_{\mathrm{GD}}=\left(\mathrm{f}_{\mathrm{GDene}}, \mathrm{f}_{\mathrm{GDcor}}, \mathrm{f}_{\mathrm{GDcon}}, \mathrm{f}_{\mathrm{GDidm}}\right) \\
& \mathrm{fL}=\left(\mathrm{f}_{\mathrm{LH}}, \mathrm{f}_{\mathrm{LV}}, \mathrm{f}_{\mathrm{LD}}\right) \\
& \mathrm{f}_{\mathrm{LH}}=\left(\mathrm{f}_{\mathrm{LHh} \_ \text {rrop }}, \mathrm{f}_{\mathrm{LHV} \_ \text {crop }}, \mathrm{f}_{\mathrm{LHc} \_ \text {crop }}\right)
\end{aligned}
$$




$$
\begin{gathered}
\mathrm{f}_{\mathrm{LV}}=\left(\mathrm{f}_{\mathrm{LVh} \_ \text {crop }}, \mathrm{f}_{\mathrm{LV} V_{\text {__crop }}}, \mathrm{f}_{\mathrm{LV} \text { __rop }}\right) \\
\mathrm{f}_{\mathrm{LD}}=\left(\mathrm{f}_{\mathrm{LDh} \_ \text {crop }}, \mathrm{f}_{\mathrm{LDV} \_ \text {crop }}, \mathrm{f}_{\mathrm{LDc} \_ \text {crop }}\right) \\
\mathrm{f}_{\mathrm{QUER} Y}=(\mathrm{fG}, \mathrm{fL})
\end{gathered}
$$

\section{Bag of Words (Bow)}

Given a set of training images, a K-means clustering algorithm is applied to cluster the regions on the basis of these features. These clusters which they call "blobs" compose the vocabulary for the set of images. Each blob is assigned a unique integer to serve as its identifier (analogous to a word's ASCII representation) [20].

All images in the training set can now be represented as a set of blobs from this vocabulary. Given a new test image, it can be segmented into regions and region features can be computed. The blob which is the closest to it in the cluster space is assigned to it. The basic idea of Bag of Words is to depict each image as an order less collection of local features. For compact representation, a visual vocabulary is usually constructed to describe BoW through the clustering of features. With the visual vocabulary, we can describe the image as a feature vector according to the presence or count of each visual word. Under the supervised learning platform, the feature vector forms the basic visual cue for object and scene classification. In a BoW approach, the classification stage turns into a histogram based classification, although the paradigm is simple, it do not contain any geometry information.

\section{E. Similarity Measurement}

The characteristic features of the query image and all the images in database are stored in terms of feature vectors. Based on these vectors the similarity or dissimilarity between the images is computed. Methods used to classify images either measure the difference or similarity between two vectors. Two vectors with small difference will have large similarity. We have used Euclidean distance which is the most common metric for measuring the distance between two vectors. Given two vectors $Q$ and $D$, where

$$
Q=\left[\begin{array}{c}
q_{1} \\
q_{2} \\
\vdots \\
q_{n}
\end{array}\right] \text { and } D=\left[\begin{array}{c}
d_{1} \\
d_{2} \\
\vdots \\
d_{n}
\end{array}\right]
$$

Then the Euclidean distance between them is given by

$$
E D=\sqrt{\sum_{i=1}^{n}\left(Q_{i}-D_{i}\right)^{2}}
$$

Where, Q represents the feature vector of query image and D represents the feature vector of database image. Lower the distance value, larger the similarity between the two images. After computing the Euclidean distance the images are sorted in the increasing order of their distance from the query image. Finally the top 50 images are displayed as relevant to the query. 

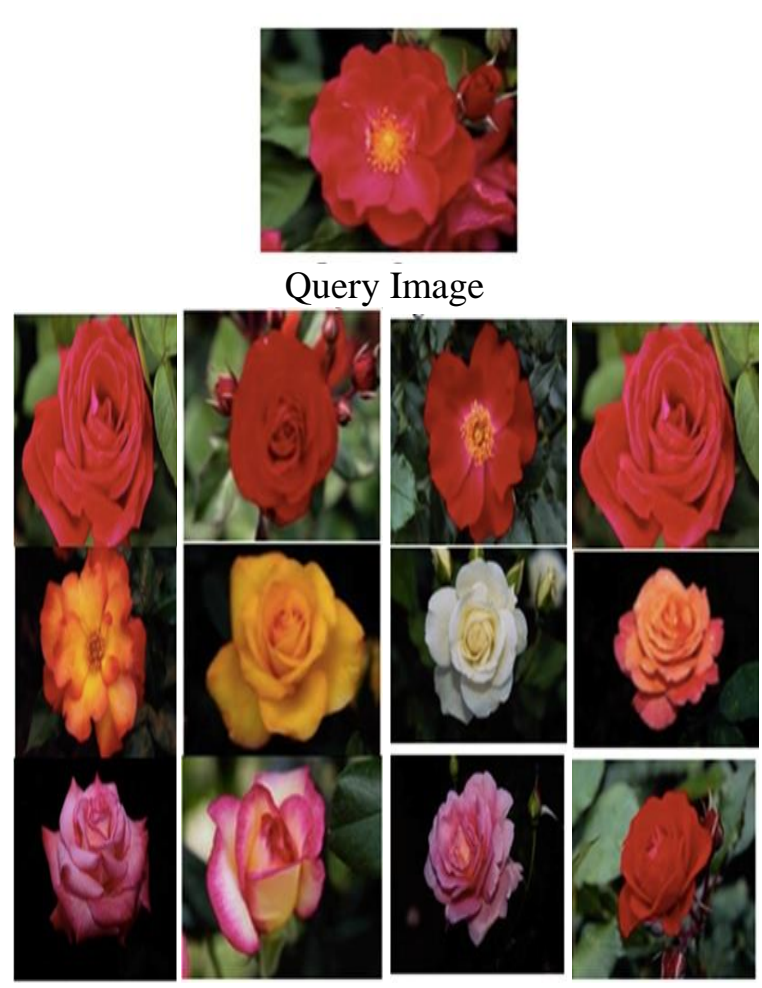

Figure 4. Query Image Class of Flower (top) and Top 12 Retrieved Images

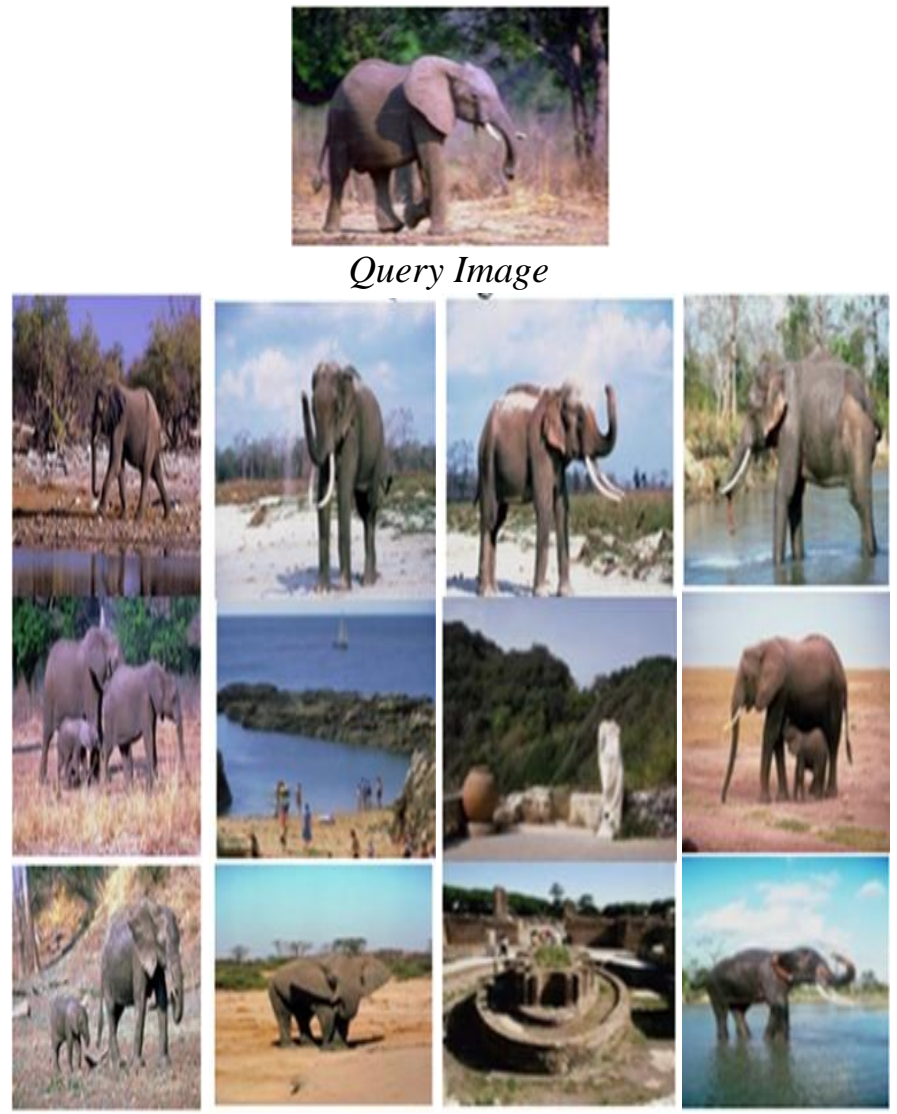

Figure 5. Query Image from Class of Sea (top) and Top 12 Retrieved Image 


\section{Proposed Algorithm}

1. Consider an image is to be $\mathrm{NxN}$ size of the array.

2. Convert rgb image into ycbcr format where $\mathrm{y}$ is luminance model, $\mathrm{cb}$ is blue color and cr is red color.

3. Take Y component for dwt transform.

4. Apply DWT transform, the image is decomposed into four frequency sub-bands, LL, $\mathrm{LH}, \mathrm{HL}$, and $\mathrm{HH}$ where L denotes low frequency and $\mathrm{H}$ denotes high frequency.

$$
\begin{aligned}
L L_{j+1}(x, y) & =\sum_{m, n} L[n] L[m] L L_{j\left(2^{j+1} m-x, 2^{j+1} n-y\right)} \\
L H_{j+1}(x, y) & =\sum_{m, n} L[n] H[m] L L_{j\left(2^{j+1} m-x, 2^{j+1} n-y\right)} \\
H L_{j+1}(x, y) & =\sum_{m, n} H[n] L[m] L L_{j\left(2^{j+1} m-x, 2^{j+1} n-y\right)} \\
H H_{j+1}(x, y) & =\sum_{m, n} H[n] H[m] L L_{j\left(2^{j+1} m-x, 2^{j+1} n-y\right)}
\end{aligned}
$$

5. Divide the image into sub-regions: horizontal, vertical and center region:

$$
\begin{aligned}
& f G=\left(f_{G H}, f_{G V}, f_{G D}\right) \\
& \mathrm{f}_{\mathrm{GH}}=\left(\mathrm{f}_{\mathrm{GHene}}, \mathrm{f}_{\mathrm{GHcor}}, \mathrm{f}_{\mathrm{GHcon}}, \mathrm{f}_{\mathrm{GHidm}}\right) \\
& \mathrm{f}_{\mathrm{GV}}=\left(\mathrm{f}_{\mathrm{GVene}}, \mathrm{f}_{\mathrm{GV} \text { cor }}, \mathrm{f}_{\mathrm{GVcon}}, \mathrm{f}_{\mathrm{GVidm}}\right) \\
& \mathrm{f}_{\mathrm{GD}}=\left(\mathrm{f}_{\mathrm{GDene}}, \mathrm{f}_{\mathrm{GDcor}}, \mathrm{f}_{\mathrm{GDcon}}, \mathrm{f}_{\mathrm{GDidm}}\right) \\
& \mathrm{fL}=\left(\mathrm{f}_{\mathrm{LH}}, \mathrm{f}_{\mathrm{LV}}, \mathrm{f}_{\mathrm{LD}}\right) \\
& \mathrm{f}_{\mathrm{LH}}=\left(\mathrm{f}_{\mathrm{LHh} \_ \text {crop }}, \mathrm{f}_{\mathrm{LHv} \_ \text {crop }}, \mathrm{f}_{\mathrm{LHc} \_ \text {crop }}\right) \\
& \mathrm{f}_{\mathrm{LV}}=\left(\mathrm{f}_{\mathrm{LVh} \_ \text {crop }}, \mathrm{f}_{\mathrm{LV} \mathrm{V}_{\text {_crop }}}, \mathrm{f}_{\mathrm{LV} \mathrm{C}_{\text {_crop }}}\right) \\
& f_{L D}=\left(f_{\text {LDh_crop }}, f_{\text {LDv_crop }}, f_{\text {LDc_crop }}\right)
\end{aligned}
$$

6. After splitting the image into sub-regions, two statistical measures are computed for each region. These measures are mean $(\mu)$ and standard deviation $(\sigma)$.

$$
\begin{array}{r}
\mu=\frac{\sum_{i=1}^{M} \sum_{j=1}^{M} f(i, j)}{M \times N} \\
\sigma=\sqrt{\frac{\sum_{i=1}^{M}(|f(i, j)|-\mu)^{2}}{M \times N}}
\end{array}
$$

Here $\mathrm{M}, \mathrm{N}$ represent the dimensions of the image and $\mathrm{f}(\mathrm{i}, \mathrm{j})$ represents the intensity value at an index $(\mathrm{i}, \mathrm{j})$.

7. Gray Level Co-occurrence Matrix (GLCM) computed over horizontal, vertical and diagonal sub-matrices.

8. Given a set of training images, a K-means clustering algorithm is applied to cluster the regions on the basis of these features.

$$
\mathrm{f}_{\mathrm{QUERY}}=(\mathrm{fG}, \mathrm{fL})
$$




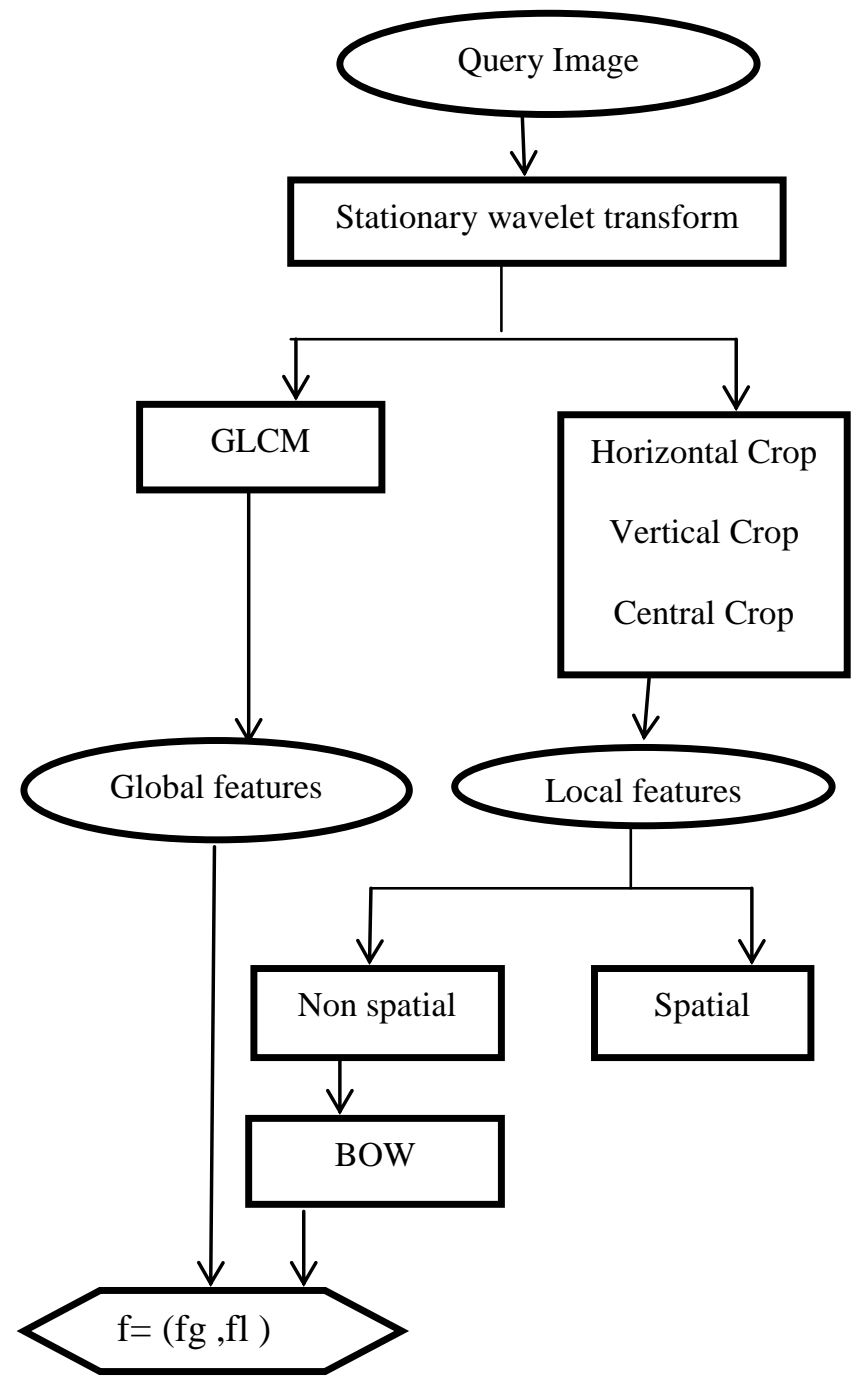

A. Performance Evaluation

As a measure of performance we have used two widely used metrics of Precision and Recall. Precision is a measure of ability of CBIR algorithm to retrieve only relevant images, while Recall decides the ability of CBIR algorithm to retrieve all relevant images as defined respectively.

$$
\begin{aligned}
& P=\frac{\text { No. of relevant image retrieved }}{\text { Total number of image retrieved }} \\
& R=\frac{\text { No. of relevant image retrieved }}{\text { number of image in the datebese }}
\end{aligned}
$$

\section{Conclusion}

In this paper, we presented technique for Content Based Image Retrieval by merging the color and texture features called Wavelet-Based Color Histogram Image Retrieval (WBCHIR).The comparison between the images is ascertained by means of a distance 
function. The proposed paper result better than the other retrieval methods in terms of average accuracy. Moreover, the computational steps are effectively reduced with the use of Wavelet transformation.

\section{References}

[1] M. Flickner, H Sawhney, W. Niblack, J. Ashley, Q. Huang, B. Dom, M. Gorkani, J. Hafne, D. Lee, D. Petkovic, D. Steele and P. Yanker, "Query by Image and Video Content The QBIC System", IEEE Computer, (1995), pp. 23-32.

[2] A. Gupta and R. Jain, "Visual information retrieval", Communications of the ACM vol. 40, no. 5, (1997), pp. 70-79.

[3] A. Pentland, R. W. Picard and S. Scaroff, "Photobook: Content-Based Manipulation for Image Databases", International Journal of Computer Vision, vol. 18, no. 3, (1996), pp. 233-254.

[4] J. R. Smith and S. F. Chang, "VisualSEEk: a fully automated content-based image query system", ACM Multimedia, (1996).

[5] J. Wang, G. Wiederhold, O. Firschein and S. We, "Content-based Image Indexing and Searching Using Daubechies' Wavelets", International Journal on Digital Libraries (IJODL), vol. 1, no. 4, (1998), pp. $311-328$

[6] C. Carson, S. Belongie, H. Greenspan and J. Malik, "Blobworld: image segmentation using expectationmaximization and its application to image querying", IEEE Trans. Pattern Anal. Mach. Intell., vol. 8, no. 8, (2002), pp. 1026-1038.

[7] J. Wang, J. LI and G. Wiederhold, "SIMPLIcity: Semantics-sensitive integrated matching for picture libraries", IEEE Transactions on Pattern Analysis and Machine Intelligence, vol. 23, no. 9, (2001), pp. 947-963.

[8] C. H. Lin, R. T. Chen and Y. K. Chan, "A smart content-based image retrieval system based on color and texture feature", Image and Vision Computing, vol. 27, (2009), pp. 658-665.

[9] N. Jhanwar, S. Chaudhurib, G. Seetharamanc and B. Zavidovique, "Content based image retrieval using motif co-occurrence matrix", Image and Vision Computing, vol. 22, (2004), pp. 1211-1220.

[10] P. W. Huang and S. K. Dai, "Image retrieval by texture similarity", Pattern Recognition, vol. 36, (2003), pp. 665-679.

[11] P. S. Hiremath and J. Pujari, "Content Based Image Retrieval based on Color, Texture and Shape features using Image and its complement", 15th International Conference on Advance Computing and Communications, IEEE, (2007).

[12] Y. Chen and J. Z. Wang, "A Region-Based Fuzzy Feature Matching Approach to Content Based Image Retrieval", IEEE Transactions on Pattern Analysis and Machine Intelligence, vol. 24, no. 9, (2002), pp. $1252-1267$.

[13] Y. Rubner, L. J. Guibas and C. Tomasi, "The earth mover's distance, multidimensional scaling, and color-based image retrieval", Proceedings of DARPA Image understanding Workshop, (1997), pp. 661668.

[14] M. B. Rao, B. P. Rao, and A. Govardhan, "CTDCIRS: Content based Image Retrieval System based on Dominant Color and Texture Features", International Journal of Computer Applications, vol. 18, no. 6, (2011), pp. 0975-8887.

[15] R. Sriram, J. M. Francos and W. A. Pearlman, "Texture coding using a word decomposition based model", IEEE Transactions of Image Processing, vol. 5 (1996), pp. 1382-1386.

[16] D. Marr and H. K. Nishihara, "Representation and recognition of the spatial organization of threedimensional shapes", Proceedings of the Royal Society of London Series B, Biological Sciences, vol. 200, (1978), pp. 269-294.

[17] M. Brady, Criteria for representations and of shape, Human and Machine Vision Academic, (1993), pp. $39-84$.

[18] W. K. Pratt, Bibliography, Digital Image Processing (third ed.), 2002, pp. 717-722. <http://dx.doi.org/10.1002/0471221325.biblio>.

[19] E. Valveny and P. Dosch, "Symbol Recognition Contest: A Synthesis, Graphics Recognition”, 2004, pp. 368-385. <http://www.springerlink.com/content/65dlhypeg3ha96ex>.

[20] Structured representations in a content based image retrieval context Romain Raveaux, Jean-Christophe Burie, Jean-Marc Ogier.

[21] R. M. Haralick, K. Shanmugam and I. Dinstein, "Textural Features for Image Classification", IEEE Transactions on Systems, Man, and Cybernetics, (1973), pp. 610-621.

[22] R. Cossu, "Segmentation by means of textural analysis", Pixel, vol. I, no. 2, (1988), pp. 21-24.

[23] R. M. Haralick, K. Shanmugam, and I. Dinstein, "Textural features for image classification", IEEE Trans. Systems, Manufact. Cybernet., vol. SMC-3, no. 6, (1988), pp. 610-621.

[24] S. Agarwal, A. K. Verma and Preetvanti Singh, "Content Based Image Retrieval using Discrete Wavelet Transform and Edge Histogram Descriptor", International Conference on Information Systems and Computer Networks, (2013), pp. 19-23. 
[25] T. Yumin and M. Lixia, "Image Retrieval Based on Multiple Features Using Wavelet" 5th IEEE International Conference on Computational Intelligence and Multimedia Applications, (2003), pp. 137142.

[26] P. S. Hiremath, S. Shivashankar and J. Pujari, "Wavelet Based Features for Color Texture classification with Application to CBIR", International Journal of Computer Science and Network Security, vol. 6, no. 9A, (2006), September.

[27] R. C. Gonzalez and R. E. Woods, "Digital Image Processing", Pearson Education, Third Edition, Copyright (C) (2008).

[28] Fusion of Local and Global Features using Stationary Wavelet Transform for Efficient Content Based Image Retrieval 2014 IEEE Students' Conference on Electrical, Electronics and Computer Science. 
International Journal of Signal Processing, Image Processing and Pattern Recognition Vol.8, No.6 (2015) 